\title{
Performance studies of Micromegas detectors for the ATLAS experiment
}

\author{
Paolo lengo* ${ }^{* \dagger}$ \\ INFN Naples \\ E-mail: paolo.iengo@cern.ch
}

\begin{abstract}
Micromegas is one of the detector technologies that have been chosen for precision tracking and trigger purposes for the upgrade of the forward muon system of the ATLAS experiment in view of the LHC luminosity increase. We present a survey of the detector performances obtained in recent test beam campaigns with high energy particle beams. Efficiency, temporal and spatial resolutions measured for the Micromegas detector configuration to be used in ATLAS are fully compatible with the requirements of the experiment upgrade.
\end{abstract}

The European Physical Society Conference on High Energy Physics -EPS-HEP2013

18-24 July 2013

Stockholm, Sweden

\footnotetext{
* Speaker.

†n behalf of the MAMMA (Muon Atlas MicroMegas Activity) Collaboration.
} 


\section{Introduction}

The Micromegas (MM) [1] is one the the detector technologies chosen for precision tracking and trigger purposes for the upgrade of the forward muon system of the ATLAS experiment in view of the LHC luminosity increase. Together with sTGC, the MM will compose the New Small Wheels (NSWs), replacing the present Small Wheels of ATLAS in 2018 [2]. Eight detector layers of Micromegas, arranged in two quadruplets, will be used to build each of the 16 sectors of the two NSWs, leading to a total active area of about $1200 \mathrm{~m}^{2}$. The layout of the detector has been optimized for the use of MM in ATLAS: $128 \mu \mathrm{m}$ amplification gap (with a mechanically floating mesh); $5 \mathrm{~mm}$ drift gap; $\sim 450 \mu \mathrm{m}$ strip pitch (with resistive strips for spark suppression [3]); gas mixture Ar: $\mathrm{CO}_{2}$ 93:7. Figure 1 shows a sketch of the MM layout and its principle of operation.
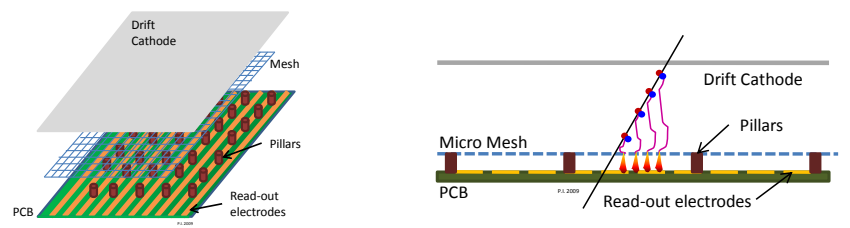

Figure 1: Sketch (not to scale) of the layout and operating principle of a Micromegas detector.

Performance of MM for ATLAS have been extensively studied at the CERN SPS H6 beam line ( $120 \mathrm{GeV} / \mathrm{c}$ pion beam, intensity of $\sim 10 \mathrm{kHz}$ on a spot of $\sim 2 \mathrm{~cm}^{2}$ ) on small resistive prototypes $\left(10 \times 10 \mathrm{~cm}^{2}\right)$ with a strip pitch of $400 \mu \mathrm{m}$. The detectors under test were mounted, in pairs, in the back-to-back configuration, similarly to what will happen in the NSWs. They were operated with the following settings: $\mathrm{HV}_{\text {drift }}=300 \mathrm{~V} ; \mathrm{HV}_{\mathrm{amp}}=500 \mathrm{~V}$. The orientation of the chambers could be adjusted to vary the impact angle of the beam from $0^{\circ}$ (beam perpendicular to the read-out plane) to $40^{\circ}$. The front-end electronics was based on the APV25 ASIC read out via the Scalable Readout System [4]. The trigger signal was provided by the coincidence of three scintillators plus a veto. Time and charge informations were obtained for each hit by a functional fit to the output signal shape of the APV25, operated at $40 \mathrm{MHz}$ in 27-sample configuration. The APV clock was not synchronized with the trigger, resulting in a time jitter of $\pm 12.5 \mathrm{~ns}$.

\section{Results}

Figure 2 left shows the distribution of the differences of the first hit arrival time of two MM traversed by the same particle. In this way the time jitter is canceled. The time resolution (given by $\sigma / \sqrt{2}$ ) results to be $10.3 \pm 0.2 \mathrm{~ns}$. The intrinsic time resolution of a Micromegas with the mentioned operating settings is expected to be even better; the quoted result includes additional contrbutions from the front-end and DAQ chain and from the offline analysis still to be optimized. However, the measured resolution is already in agreement with the requirements of the NSW.

For perpendicular tracks, the spatial resolution measured with the cluster centroid method resulted to be of the order of $70 \mu \mathrm{m}$. For impact angles $>10^{\circ}$ the same method does not provide the desired resolution. In this case the $\mu \mathrm{TPC}$ mode [5] can be exploited to perform a local track 

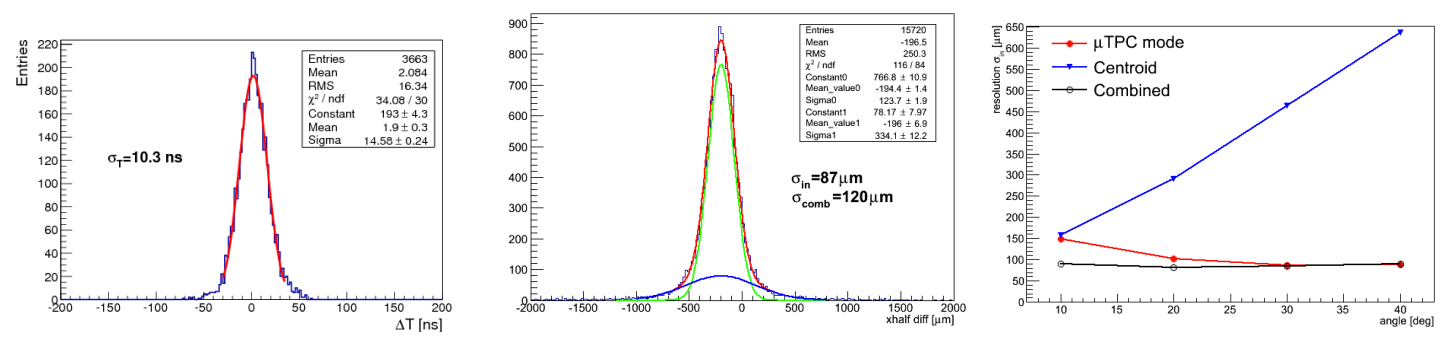

Figure 2: Left: distribution of first hit time differences for a Micromegas pair. Center: distribution of $x_{\text {half }}$ differences for a Micromegas pair. Right: MM spatial resolution as a function of the track impact angle measured with the charge centroid method (blue points), $\mu$ TPC method (red points) and by combining the two methods (black points).

reconstruction in the drift gap, with the vertical coordinate of the hits reconstructed from the time measurement. The $x$ coordinate of the track is measured at the half of the drift gap ( $\left.x_{\text {half }}\right)$. Figure 2 center shows the distribution of $x_{\text {half }}$ difference between a MM pair for an impact angle of $30^{\circ}$, with a double gaussian fit. The single plane resolution results to be $87 \mu \mathrm{m}$ when only the dominant gaussian is considered and $120 \mu \mathrm{m}$ by taking a weighted average of the two gaussians. Figure 2 right shows the spatial resolution measured as a function of the track impact angle with the cluster centroid (blue points) and the $\mu$ TPC (red points) methods. By combining the two methods we can achieve an uniform spatial resolution below $100 \mu \mathrm{m}$ for all impact angles up to $40^{\circ}$ (black points).

Chamber inefficiencies have been also measured, resulting in a global inefficiency in the range 1 to $2 \%$, consistent with the partially dead area due to the presence of $300 \mu \mathrm{m}$ diameter pillars, with pitch spacing of $2.5 \mathrm{~mm}$.

\section{Conclusions}

Performance of Micromegas detectors for the ATLAS upgrade have been studied at the CERN SPS beam line. Chamber efficiency, temporal and spatial resolutions are fully compatible with the requirements of the ATLAS New Small Wheels. In particular, the exploitation of the $\mu$ TPC operational mode allows to reach, in combination with the charge centroid method, an uniform spatial resolution below $100 \mu \mathrm{m}$ for all impact angles of the NSW. After a long R\&D effort, the MM technology is now mature to equip large detector systems in the harsh environment of LHC.

\section{References}

[1] Y. Giomataris et al., Nucl. Instr. Meth. A 376 (1996) 29-35.

[2] ATLAS Collaboration, New Small Wheel Technical Design Report, CERN-LHCC-2013-006.

[3] T. Alexopoulos et al., Nucl. Instr. Meth. A 640 (2011) 110.

[4] S. Martoiu, H. Muller, J. Toledo, (NSS/MIC), 2011 IEEE; M.J. French, et al., Nucl. Instr. Meth. A 466 (2001) 359.

[5] P. Iengo et al., Nucl.Instrum.Meth. A617 (2010) 161-165 